\title{
Importance of the Location of Myocardial Infarc- tion and Ventricular Aneurysm on Left Ventricular Function
}

\author{
Katsuo Ozawa, M.D.
}

\section{SUMMARY}

This experimental study was performed to evaluate the possibility that the site per se of infarction or ventricular aneurysm after acute myocardial infarction had a unique important role in left ventricular function.

Acute myocardial infarction and ventricular aneurysm were made in either the anterior or the inferior wall of the left ventricle using 24 dogs. Infarction was made by the multiple ligation method. The weight of the infarcted area was $23.1 \pm 3.9 \%$ of the left ventricular free wall in the anterior wall group (12 dogs), and was $23.8 \pm 4.8 \%$ in the inferior wall group (12 dogs). Ventricular aneurysm was made of right atrial homograft. The capacity of ventricular aneurysm, measured by casts, was $37.8 \pm 7.7 \%$ of the left ventricular cavity in the anterior wall group and was $38.3 \pm 7.3 \%$ in the inferior wall group. There was no significant difference in either the size of infarction or the capacity of ventricular aneurysm between 2 groups $(p=0.05)$.

Ventricular function curves were obtained before infarction (control), after infarction, and after the creation of ventricular aneurysm, plotting left ventricular stroke work (LVSW) per body weight against left atrial mean pressure (LAMP). The change in ventricular function curves after infarction and the creation of ventricular aneurysm was not different significantly between 2 groups.

In the anterior wall group, LVSW/LAMP decreased to $47.8 \pm 9.8 \%$ of the control after infarction, and further to $27.1 \pm 6.9 \%$ after the creation of ventricular aneurysm. In the inferior wall group, it lowered to $47.6 \pm$ $12.5 \%$ and again to $26.5 \pm 6.4 \%$, respectively. There was no difference in these values between 2 groups statistically $(p=0.05)$.

From the result of this experiment, it was concluded that the impairment of left ventricular function was equal between these 2 groups not only after infarction but also after the creation of ventricular aneurysm and that the anterior wall would not have more important contribution to the pumping ability of the left ventricle as compared with the inferior wall.

From the First Department of Surgery, University of Nagoya, School of Medicine, 65 Tsurumaicho, Showa-ku, Nagoya 466, Japan. (Director: Prof. Yohtaro Iyornasa, M.D.)

Received for publication May 20, 1977. 


\section{Additional Indexing Words :}

LVSW Left atrial mean pressure Volume loading Ventricular function curve

A $S$ a result of improved method of patient care, immediate diagnosis and treatment of life-threatening arrhythmias, death from arrhythmias complicating acute myocardial infarction has become less common. However, power failure of the heart and cardiogenic shock still remain the important mortality at present.

Although the degree of impaired function of the left ventricle is usually greater in anterior than inferior wall infarction, and cardiogenic shock develops more frequently in patients with acute infarction of the anterior wall than of the inferior one, the possibility that the anterior wall is unique in contribution to left ventricular performance is controversial. Major determinants of hemodynamic consequence of acute myocardial infarction such as extent, site and reserve of remaining viable mass, which mainly depend upon the state of coronary arteries, are so varied in degree that their isolated contribution to pump dysfunction is very difficult to evaluate in clinical cases.

The following experiment in dogs was performed to study the possibility that the location per se of infarction and ventricular aneurysm was related to the degree of left ventricular dysfunction in acute infarction and aneurysm of either the anterior or the inferior wall of the left ventricle.

\section{METHODS}

Twenty-four adult mongrel dogs of both sexes, weighing 10 to $30 \mathrm{Kg}$, were anesthetized with intravenously administered sodium thiopental of $25 \mathrm{mg} / \mathrm{Kg}$ and ventilated through an endotracheal tube with a respirator (MARK 7, Bird Co. Ltd.). A polyethylene catheter $(6 \mathrm{~F})$ was introduced in a retrograde fashion from the femoral artery into the aorta. A Swan-Ganz Flow Directed Thermodilution Catheter (Model No. 93 A-118-7F, Edwards Lab.) was inserted into the femoral vein and advanced to the pulmonary artery under pressure monitoring. Electrocardiogram (ECG) was monitored in standard limb leads. Thoracotomy was made through the left 5 th intercostal space on the right lateral position and the pericardial cradle was created to support the exposed heart. Another polyethylene catheter (6F) was placed into the left atrium through a small branch of the pulmonary vein. Aortic and left atrial mean pressures were recorded through transducers (TMI, BLH, MPU-0.5-290-0-III) on a 4-channel recorder (Multipurpose polygraph RH150, Nihon Kohden). The zero reference point was at the level of the right atrium. Respiration was temporarily suspended by opening the airway to ambient pressure during recording. Cardiac output was determined by the thermodilution method with the use of a cardiac output computer (Model 9500, Edwards Lab.). Fig. 1 shows the schema of this preparation. Animals were divided into 2 groups, anterior 


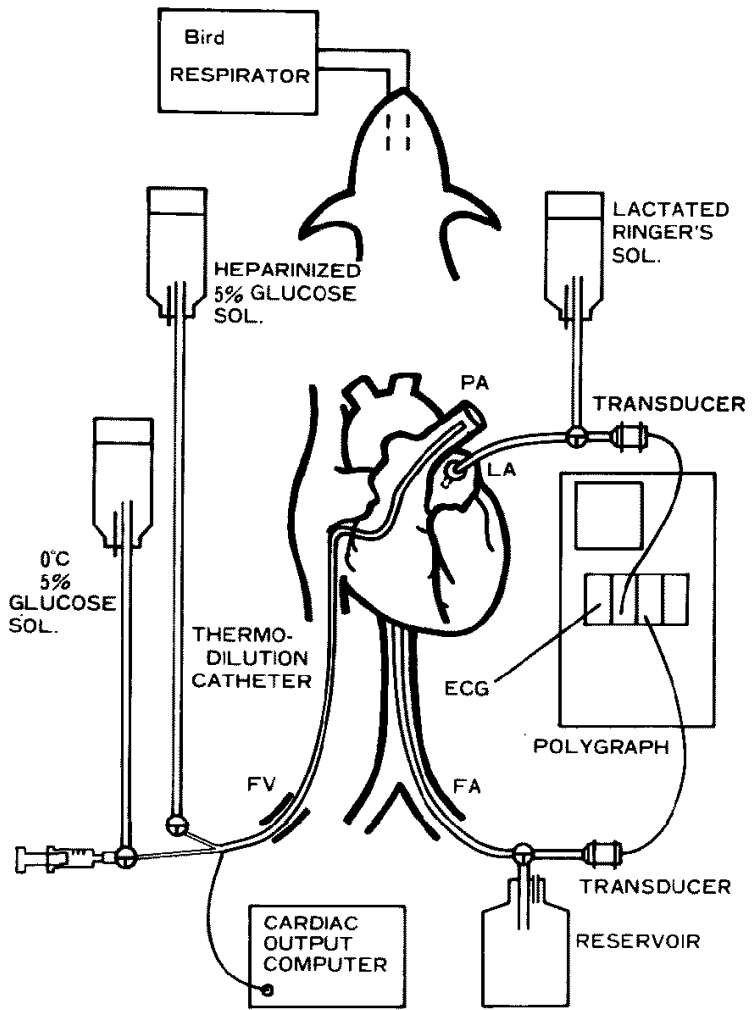

Fig. 1. Schema of the open chest preparation in this experiment.

and inferior wall group.

Anterior wall group: Twelve dogs comprised this group. The anterior descending artery distal to the first marginal branch was ligated, together with interconnecting coronary arteries, while a fresh homologous right atrial graft obtained from a little smaller donor dog was sutured on the anterior wall of the left ventricle (Fig. 2A, Fig. 3A). Then, anterior wall infarction was made.

Inferior wall group: Twelve dogs were included in this group. Inferior wall infarction was made by ligating the posterior descending branch of the left circumflex artery 1 to $1.5 \mathrm{~cm}$ distal to its origin together with interconnecting coronary arteries, while the same kind of graft was sutured on the inferior wall of the left ventricle (Fig. 2B).

In each animal, ventricular aneurysm was created by removing a core of the myocardium, about $1 \mathrm{~cm}$ in diameter, from the area within the confines of the sutured graft with a puncher, which was introduced from the vena cava of the grafted right atrium (Figs. 3, 4). Cardiac output, heart rate, aortic, and left atrial mean pressures were measured. The measurement was done before and after volume loading in 3 stages: prior to infarction (control), after infarction and after the creation of ventricular aneurysm. The volume loading was made by a rapid intraatrial injection of 100 to $200 \mathrm{ml}$ of lactated Ringer's solution in the initial stage. Then, an adequate amount of blood was extracted to return the left atrial mean 
870

OZAWA

Jap. Heart J.
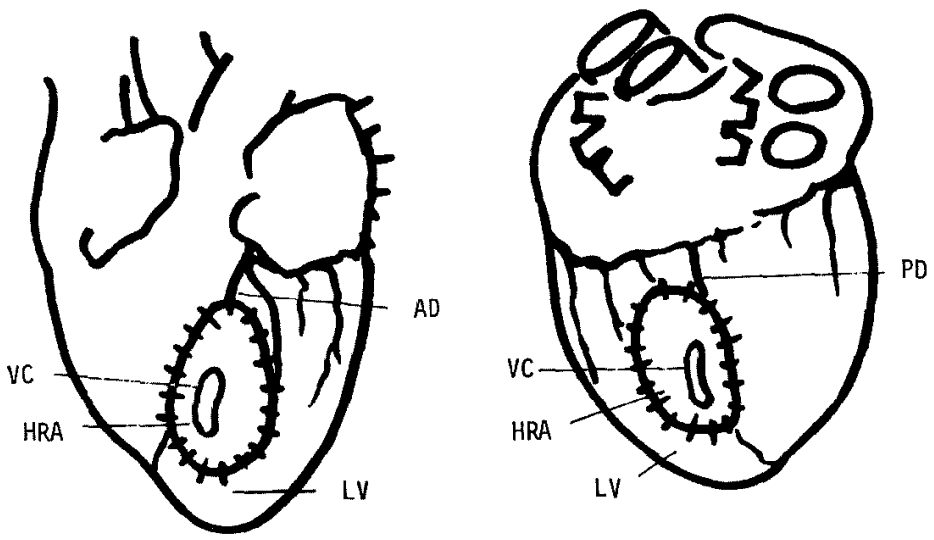

Fig. 2. The location of infarction following the ligation of the anterior descending branch (AD) in the anterior wall group (A) and the posterior descending branch (PD) in the inferior wall group (B), in the course of grafting the homologous right atrium (HRA) as a sac of ventricular aneurysm. VC, the vena cara of the grafted right atrium.
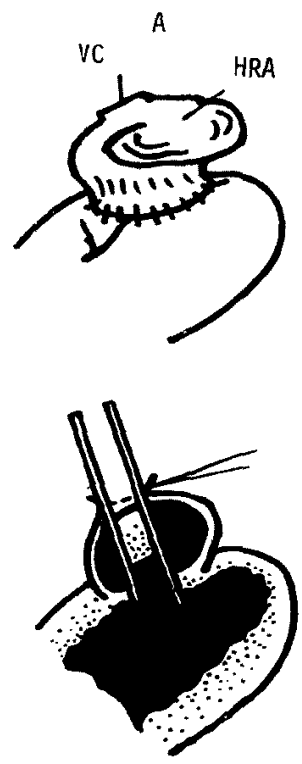

C
B

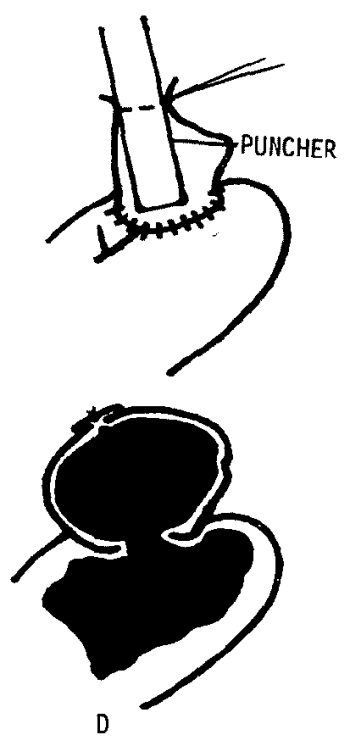

Fig. 3. Technique employed for the creation of ventricular aneurysm. A, the right atrium of a donor dog was grafted on the left ventricular wall as a sac of ventricular aneurysm. B, a puncher (metal cylinder with a circular blade) was inserted through the vena lava (VC) of the graft (HRA). C, the myocardium was punched out and a communication to the left ventricular cavity was made. D, a homologous right atrial left ventricular aneurysm was created. 


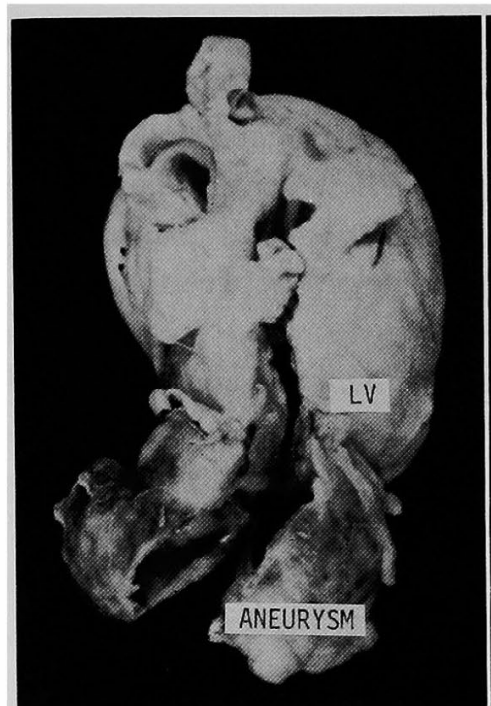

Fig. 4 .

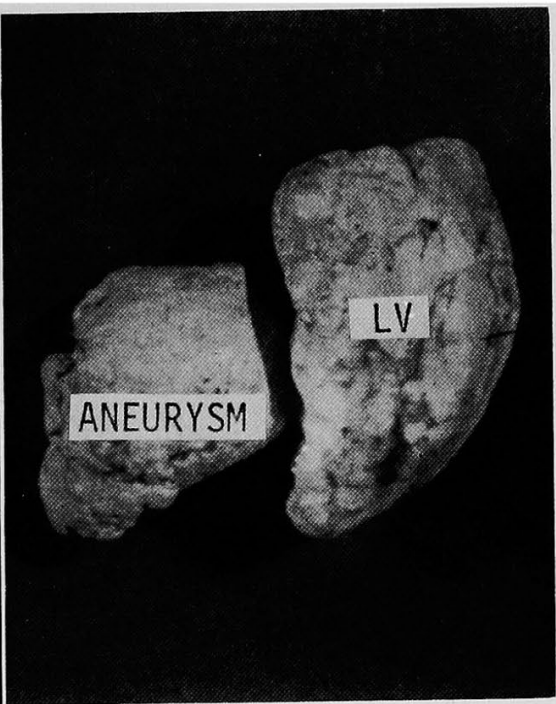

Fig. 5.

Fig. 4. A specimen of ventricular aneurysm. This dog survived 10 days after the creation of ventricular aneurysm.

Fig. 5. Casts of the left ventricular cavity and ventricular aneurysm of the inferior wall group.

pressure as close to the initial level as possible, and was stored in a reservoir after heparinization. This blood was reserved for the subsequent volume loading.

Left ventricular function curves were composed by plotting left ventricular stroke work (LVSW) per body weight in kilogram against left atrial mean pressure in 3 stages. Left ventricular stroke work was calculated as follows,

$$
\mathrm{LVSW}=(\text { AMP }- \text { LAMP }) \times \mathrm{SV} \times 1.36 \times 0.01
$$

where $\mathrm{LVSW}=$ left ventricular stroke work in gram-meter/sec

$\mathrm{AMP}=$ aortic mean pressure in $\mathrm{mmHg}$

$\mathrm{LAMP}=$ left atrial mean pressure in $\mathrm{mmHg}$

$\mathrm{SV}=$ stroke volume or cardiac output $(\mathrm{ml} / \mathrm{min}) /$ heart rate.

LVSW/LAMP was calculated after infarction and after the creation of ventricular aneurysm in each animal and expressed in per cent of the control.

After all hemodynamic measurements were done, the animal was sacrificed by injecting $20 \mathrm{ml}$ of $\mathrm{KCl}$ solution into the left atrium. The left ventricle with the aneurysm was casted. Their volumes were measured from these casts (Fig. 5). Then, the infarcted area of the left ventricle was resected and weighed.

\section{RESULTS}

Production of acute myocardial infarction resulted in ventricular fibrillation in 2 cases of each group. All of them were successfully reversed with electroversion allowing continuation of the experiment. The weight of the in- 
farcted area was $23.1 \pm 3.9 \%$ of the left ventricular free wall in the anterior wall group, and was $23.8 \pm 4.8 \%$ in the inferior wall group. The difference between 2 groups was not significant statistically $(p=0.05)$. Ventricular aneurysm had the capacity of $37.8 \pm 7.7 \%$ of the left ventricular cavity in the anterior wall group, and $38.3 \pm 7.3 \%$ in the inferior wall group. There was no difference in relative size of ventricular aneurysm between 2 groups statistically $(p=0.05)$.

Left ventricular function curves shifted downward and to the right after
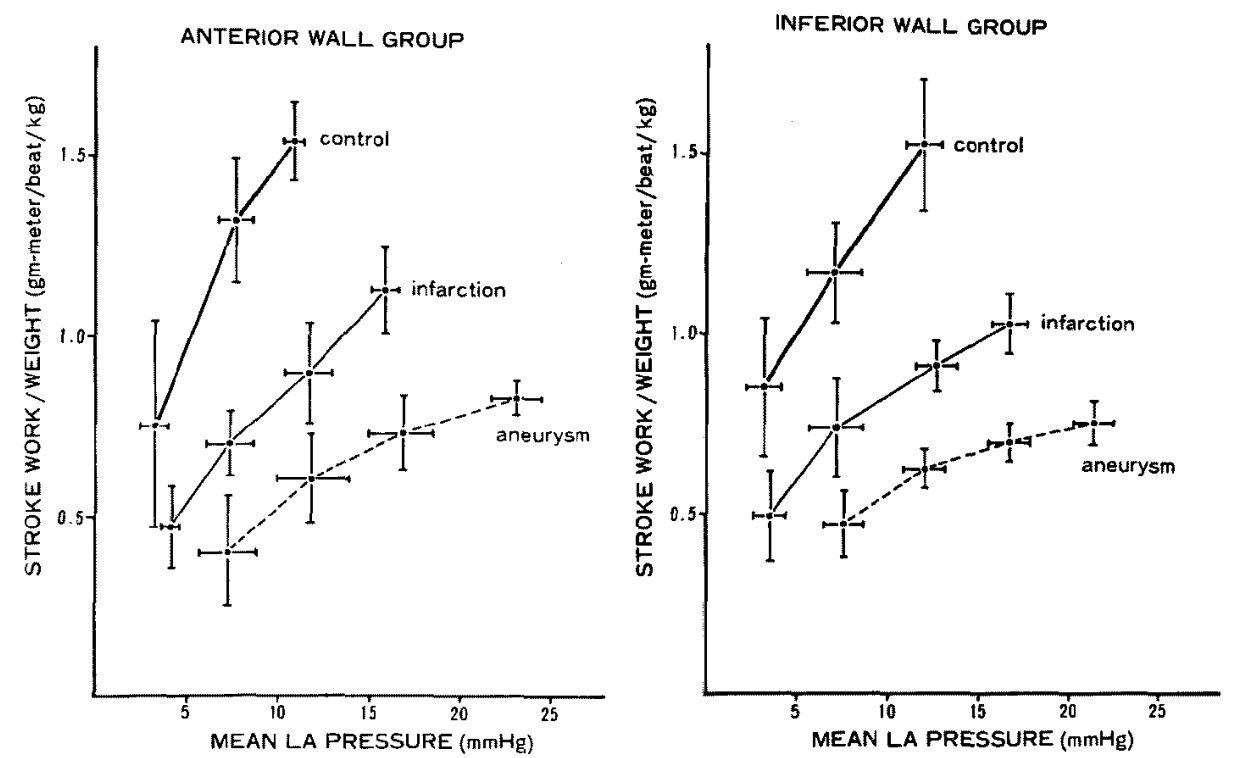

Fig. 6. Changes in left ventricular function curves of the anterior and the inferior wall group.

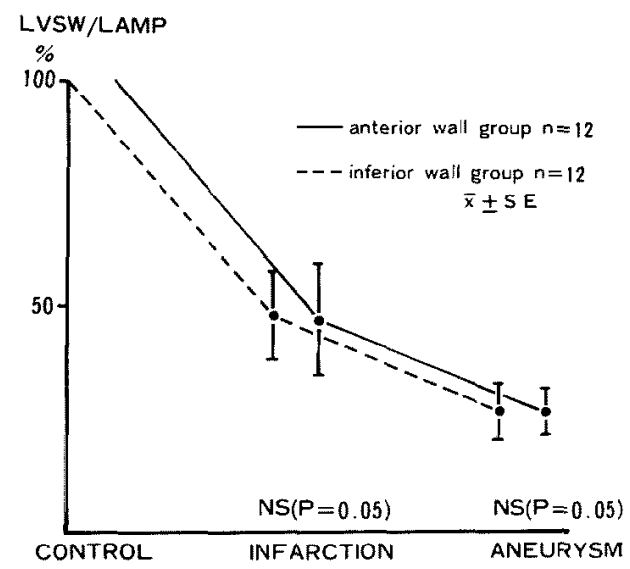

Fig. 7. Changes in left ventricular stroke work / left atrial mean pressure (LVSW/LAMP) of 2 groups. 
infarction, and further displacement to the same direction was observed after the creation of ventricular aneurysm. There was no significant difference in the degree of shifting between 2 groups, either after infarction or after the creation of ventricular aneurysm (Fig. 6).

LVSW/LAMP, expressing the average slope of each ventricular function curve, decreased to $47.8 \pm 9.8 \%$ of the control in the anterior wall group and to $47.6 \pm 12.5 \%$ in the inferior wall group, after infarction. After the creation of ventricular aneurysm, it lowered to $27.1 \pm 6.9 \%$ of the control in the anterior wall group and to $26.5 \pm 6.4 \%$ in the inferior wall group. The change in LVSW/LAMP from the control was not different statistically between 2 groups either after infarction or after the creation of ventricular aneurysm ( $\mathrm{p}=0.05)$ (Fig. 7).

\section{Discussion}

Several aspects concerning the model for infarction and ventricular aneurysm in this experiment require comments. There are many terms specifying the location of infarction such as anterior, anteroseptal, anterolateral, apical, inferior, posteroinferior, diaphragmatic, posterior, posterobasal, and so on. The accuracy of these distinctions still remains unsettled. This experiment was performed in open chest preparation and exposed heart was supported by the pericardial cradle. Because the heart would be somewhat displaced from the natural position, the ECG diagnosis of the site and the extent of infarction seemed questionable in this type of experiment. Therefore, "anterior" and "inferior" were not used in the strict criterion of ECG interpretation in this experimental model. The location described here by "inferior" would be otherwise diaphragmatic or posteroinferior portion of the left ventricle (Fig. 2B).

The multiple ligation method is considered most reliable and reproducible in making experimental infarction in canine heart. In dogs, $75 \%$ of the ventricular septum is supplied by the anterior septal artery and the remaining $25 \%$ is nourished by deep perforators from the anterior and posterior descending artery. ${ }^{1,2)}$ The level of the most proximal ligation on the anterior descending artery was distal to the origin of the anterior septal artery. Therefore, the extent of septal involvement after the multiple ligation in these 2 groups was considered equal.

The shape of ventricular aneurysm was sac-like (Fig. 4). Though the caliber of the communication to the left ventricle was small, the paradoxical expansion of ventricular aneurysm with each systolic contraction was observed in all cases. The capacity of ventricular aneurysm was large enough as com- 
pared with that of the left ventricle, but it did not impair ventricular performance so much that animals could not survive the experiment. This was proved with a fact that other 5 dogs which had not been sacrificed after this series of experiment survived more than 1 week.

Orias was the first who reported the dynamic change in the canine heart following the ligation of the anterior descending artery. ${ }^{3}$ Tennant observed a marked expansion of the ischemic region after coronary occlusion, and suggested that a considerable fraction of the total pressure developed was lost in producing such an expansion. ${ }^{4}$ Murray and Heimbecker connected paradoxical expansion of acutely infarcted myocardium with decreased cardiac output and low blood pressure. ${ }^{5), 6)}$ Herman described in a group of patients that ventricular asynergy, local disturbance in ventricular wall motion which disrupted the normal pattern of left ventricular contraction, represented an important cause of cardiac failure." Pairolero reported the hemodynamic effect of experimental cardiac akinesis in canine heart and showed that the impairment of left ventricular function was related to the size of the scar. ${ }^{8}$ ) Experimental cardiac aneurysm was studied by Austen for the first time, who observed fall of left ventricular function following the creation of ventricular aneurysm and return of it after clamping of the aneurysm at its base. ${ }^{\text {" }}$ Tyson made a study of hemodynamic effect of artificially constructed left ventricular aneurysm on left ventricular function in dogs. ${ }^{10)}$ In that study, left ventricular performance of animals which had expansile aortic homologous ventricular aneurysm was impaired more markedly than that of dogs which had relatively non-expansile Teflon ventricular aneurysm. Vayo and Klein made a theoretical analysis of left ventricular aneurysm and showed that if approximately 20 to $25 \%$ of the left ventricular area was inactivated by any pathological process, the degree of shortening distance required of myofibrils to maintain stroke volume exceeded physiological limits. ${ }^{11,12)}$ Ventricular aneurysm produces mechanical disadvantage not only by loss of contractile tissue but also by paradoxical expansion which further reduces forward stroke volume and ejection fraction. ${ }^{13), 14)}$ The dilatation of the heart to maintain adequate stroke volume adversely affects ventricular performance because increased size produces an increase in wall stress and oxygen demand by the law of LaPlace. ${ }^{15)}$ Hemodynamic importance of the size and compliance of ventricular aneurysm was also suggested by the remarkable postoperative improvement of left ventricular function in patients undergoing aneurysmectomy. ${ }^{16)-18)}$

The location itself of infarction is obviously important in relation to involvement of specific structures such as the mitral valve apparatus, the interventricular septum and the conduction system. ${ }^{19}$. When those important structures are intact and the state of coronary arteries is equal, whether there 
is any difference in left ventricular dysfunction between anterior and inferior wall infarction of the same size, is a question. In clinical studies, hemodynamic abnormality associated with anterior myocardial infarction has been reported to be severer than that of inferior myocardial infarction. ${ }^{20)-23)}$ But, in clinical cases, the extent of infarction and associated coronary artery diseases varied so much in degree. ${ }^{24)}$ Generally, the septal involvement of acute myocardial infarction is severer in anterior infarction than in inferior one. This is explained by the blood supply of the human interventricular septum. Seventy-five to $90 \%$ of blood supply of the human interventricular septum is derived from branches of the anterior descending artery. ${ }^{25)}$ Anterior myocardial infarction develops in the occlusive disease of the anterior descending artery and usually is complicated with the lesion of septal arteries.

Herman and Kong showed in the human heart that there was greater segmental shortening during systole of the anterior wall as compared with the inferior wall.7,26) Then, Zaret deduced that anterior wall contraction should have a more important contribution to left ventricular ejection, and that infarction of the anterior wall might therefore produce greater impairment than would result from the equivalent loss of inferior wall infarction. ${ }^{28}$ ) However, if the shape of the left ventricle is considered, a difference in curvature between the anterior and the inferior wall is noted. The anterior wall is more convex than the inferior wall, so it may take more concentric movement during systole. ${ }^{29)}$ The magnitude of excursion of any region of the left ventricle may not necessarily relate to the role played in ventricular ejection. In contrast to Zaret's speculation, Miller demonstrated in patients carefully matched for similar extent and pattern of dyssynergy confined to either the anterior or the inferior ventricular wall that the location of dyssynergy was unimportant as an isolated determinant of left ventricular performance in coronary artery diseases. ${ }^{30)}$ The result of this experiment also showed that the contribution of the anterior wall to left ventricular function was equal to that of the inferior wall. The location itself of left ventricular aneurysm did not play an important role either.

As for the mechanism of developing of ventricular aneurysm in the infarcted myocardium, Lowe made an analysis of the distortion expected to occur after infarction of various types and sizes. ${ }^{30}$ ) The study showed that 2 important factors determining the distortion produced in the wall of the left ventricle were the proportion of the thickness of the wall infarcted and the size of the area destroyed. From the law of La-Place and the hypothesis of Woods, the more convex anterior wall of the left ventricle would be thinner than the inferior wall which is more flat. ${ }^{15), 31)}$ On the view point of blood supply of the human heart, the inferior wall of the left ventricle has the ad- 
vantage of dual blood supply, from the circumflex branch of the left coronary artery and from the posterior descending branch of the right coronary artery, in contrast to the anterior wall which is nourished only by the anterior descending branch of the left coronary artery. Therefore, massive transmural necrosis is unlikely to occur in the inferior wall infarction. The speed of thinning and bulging of the infarcted area may be slow in the inferior wall because it may be supported by less compliant diaphragmatic portion of the pericardium, though this was not proved in this acute experimental model. These factors may be probable causes of relatively rare occurrence of inferior ventricular aneurysm. ${ }^{32)-34}$

\section{ACKNOWLEDGEMENTS}

Grateful acknowledgment is made to Prof. Iyomasa for his constant guidance in this study. The author is indebted to Dr. Yamada, Prof. of the Research Institute of Environmental Medicine, Nagoya, for valuable advise and criticism. Thanks are tendered to Dr. Tsuchioka and other members of the 1st Dept. of Surgery for helpful discussions. Thanks are also due to Dr. Takao, Prof. of Surgery, Aichi Medical University, for providing room and facilities, and to Dr. Akiyama for much assistance.

\section{REFERENCES}

1. Bertho $\mathbf{E}$, Gagnon G: A comparative study in three dimension of the blood supply of the normal interventricular septum in human, canine, bovine, porcine, ovine and equine heart. Dis Chest $46: 251,1964$

2. Blair E: Anatomy of the ventricular coronary arteries in the dog. Circulat Res 9:333, 1961

3. Orias $\mathrm{O}$ : The dynamic changes in the ventricles following ligation of the ramus descendens anterior. Am J Physiol 100: 629, 1933

4. Tennant R, Wiggers CJ: The effect of coronary occlusion on myocardial contraction. Am J Physiol 112: 351, 1935

5. Murray DWG: Pathophysiology of cause of death from coronary thrombosis. Ann Surg 126: 523,1947

6. Heimbecker RO, Chen C, Hamilton N, Murray DWG: Surgery of massive myocardial infarction. An experimental study of emergency infarctectomy. Surgery $61: 51,1967$

7. Herman MV, Gorlin R: Implications of left ventricular asynergy. Am J Cardiol 23: 538, 1969

8. Pairolero PC, McCallister BD, Hallermann FJ, Titus JL, Ellis FH: Experimental left ventricular akinesis. Results of excision. J Thorac Cardiovasc Surg 60: 683, 1970

9. Austen WG, Tsunckawa T, Bender HW, Ebert PA, Morrow AG: The acute hemodynamic effects of left ventricular aneurysm. An experimental study in dogs. J Surg Res 2: 161, 1962

10. Tyson K, Mandelbaum I, Shumacker HB Jr: Experimental production and study of left ventricular aneurysms. J Thorac Cardiovasc Surg 44: 731, 1962

11. Vayo HW: The theory of the left ventricular aneurysms. Bull Math Biophys 28: 363, 1966

12. Klein MD, Herman MV, Goplin R: A hemodynamic study of left ventricular aneurysm. Circulation 35: 614, 1967

13. Parmley WW, Chuck L, Kivowitz G, Matlo JM, Wan HJC: In vitro length-tension rela- 
tions of human ventricular anurysms. Relation of stiffness to mechanical disadvantage. Am J Cardiol 32: 889, 1973

14. Swan HJC, Forrester JS, Diamond G, Chatterjee K, Parmley WW: Hemodynamic spectrum of myocardial infarction and cardiogenic shock. A conseptual model. Circulation 45: 1097, 1972

15. Burton AG: The importance of the shape and size of the heart. Am Heart J 54: 801, 1957

16. Baxley WA, Reeves, $\mathrm{TJ}$ : Abnormal regional myocardial performance in coronary artery disease. Progr Cardiovasc Dis 13: 405, 1971

17. Cooley DA, Hallman GL: Surgical treatment of left ventricular aneurysm. Experience with excision of postinfarction lesions in 80 patients. Progr Cardiovasc Dis 11: 222, 1968

18. Kitamura S, Echevarria M, Kay JH, Krohn BG, Redington JV, Mendez A, Zubiate P, Dunne EF : Left ventricular performance before and after removal of the noncontractile area of the left ventricle and revascularization of the myocardium. Circulation 45: 1005, 1972

19. Mundth ED, Buckley MJ, Daggett WM, Sanders CA, Austen WG: Surgery for complications of acute myocardial infarction. Circulation 45: 1279, 1972

20. Moraski RE, Mantle JA, Rogers WG, Rackley CE, Russel RO Jr: Abnormally contracting segment size following anterior and inferior myocardial infarction and its relationship to left ventricular function. Twenty-Fourth Annual Scientific Session, American College of Cardiology, Houston, Texas, 1975; Am J Cardiol 35: 157, 1975 (Abstr)

21. Hamby RI, Hoffman I, Hilsenrath J, Aintablian A, Shanies $\mathrm{S}$, Padmanabhan VS: Clinical, hemodynamic and angiographic aspects of inferior and anterior myocardial infarctions in patients with angina pectoris. Am J Cardiol 34: 513, 1974

22. Rackley CE, Russel RO Jr: Left ventricular function in acute myocardial infarction and its clinical significance. Circulation 45: 231, 1972

23. Russel RO Jr, Hunt D, Rackley CE: Left ventricular hemodynamics in anterior and inferior myocardial infarction. Am J Cardiol 32: 8, 1973

24. Williams DO, Amsterdam EA, Miller RR, Mason D'T: Functional significance of coronary collateral vessels in patients with acute myocardial infarction. Relation to pump performance, cardiogenic shock and survival. Am J Cardiol 37: 345, 1976

25. Ratshin RA, Massing GK, James TN: The clinical significance of the location of acute myocardial infarction. in Myocardial Infarction, ed Cordey E, Swan HJC, The Williams and Wilkins Co, Baltimore, p. 77, 1973

26. Kong Y, Morris JJ, McIntosh HD: Assessment of regional myocardial performance from biplane coronary cineangiograms. Young Investigators' Awards Competition, The Rivergate, New Orleans, 1970; Am J Cardiol 25: 374, 1970 (Abstr)

27. Zaret BL, Pitt B, Ross RS: Determination of the site, extent and significance of regional ventricular dysfunction during acute myocardial infarction. Circulation 45: 441, 1972

28. McDonald IG: The shape and movements of the human left ventricle during systole. A study by cineangiography and by cineradiography of epicardial markers. Am J Cardiol 26: 221, 1970

29. Miller RR, Olson HG, Vismara LA, Bogren HG, Amsterdam EA, Mason DT: Pump dysfunction after myocardial infarction. Importance of location, extent and pattern of abnormal left ventricular segmental contraction. Am J Cardiol 37: 340, 1976

30. Lowe TE, Love ER: Cardiac aneurysms. A mechanical analysis of their formation. Aus J Exp Biol Med Sci 26: 497, 1948

31. Woods RH: A few applications of a physical theorem to membranes in the human body in a state of tension. J Anat Physiol 26: 362, 1892

32. Cheng TO: Incidence of ventricular aneurysm in coronary artery disease. An angiographic appraisal. Am J Med 50: 340, 1971

33. Loop FD, Effler DB, Webster JS, Groves LK: Posterior ventricular aneurysms. Etiologic factors and results of surgical treatment. New Eng J Med 288: 237, 1973

34. Loop FD, Effler DB, Navia JA, Sheldon WC, Groves LK: Aneurysm of the left ventricle. Survival and results of a ten-year surgical experience. Ann Surg 178: 399, 1973 